\title{
A Data Rate Maximization Algorithm for Multiuser OFDM Systems
}

\author{
Zeljko Ilic, and Alen Bazant
}

\begin{abstract}
In this paper, we present suboptimal integer bit allocation algorithm that maximizes overall data rate in multiuser orthogonal frequency division multiplexing (OFDM) systems implemented in wireless networks.

Assuming knowledge of the instantaneous channel gains for all users, we propose a new multiuser OFDM loading algorithm, with constrains, that maximizes the total data rate in downlink transmission. This is done by solving the maximization problem in two steps. First step provides subcarrier assignment to users based on users' requests on quality of service $(\mathrm{QoS})$ expressed in terms of a minimum signal-to-noise ratio (SNR) on each subcarrier. The second step provides bit and power allocation to subcarriers in order to maximize overall data rate. In order to reduce the computational complexity of the proposed problem we propose a simple method, in the form of a theorem, which assigns subcarriers to users and distributes bits and power among them.

We have tested the proposed algorithm in a multiuser environment for various subcarrier SNR values requested by users, and for various levels of interference in the OFDM system.

The results show that our loading method obtains a better data rate in some situations than the algorithm described in [5], which states that the data rate of a multiuser OFDM system is maximized when each subcarrier is assigned to only one user. Results show that the total number of bits per OFDM symbol or data rate grows with the number of users in the system.
\end{abstract}

Index Terms - loading algorithm, subcarrier assignment, OFDM, multiuser environment.

\section{INTRODUCTION}

An OFDM system, [7], implemented in a multiuser environment demands optimal power and bit distribution as well as optimal distribution of subchannels between users in order to achieve maximum data rate. The algorithm that adaptively allocates transmit power and bits for each subchannel in the multicarrier system is known as a loading algorithm

Numerous loading algorithms have been developed for OFDM systems. Most of them have been developed for a

Manuscript received June 29, 2004; revised June 28, 2005, August 30,2005 , and October 22, 2005. This research was supported in part by Ministry of Science and Technology, Croatia, in 2004. This paper was presented in part at the Conference on Software, Telecommunications and Computer Networks (SoftCOM) 2004.

Z Ilic and A. Bazant are with University of Zagreb, Faculty of Electrical Engineering and Computing, Zagreb, Croatia (e-mail: \{zeljko.ilic, alen.bazant\}@fer.hr). single user environment [1], [2], [3], [8], [9]. These methods are both suboptimal and computationally efficient [1]-[3], [9] or optimal but slow in delivering the power and bit allocation [8]. In a single user environment the major goal is to distribute more power in subchannels having the best channel gain. This, of course, assumes a perfect knowledge of the channel state in a transmitter. The same principle can be applied in a multiuser environment. However, in that case, there is a conflict between users who have a good channel gain in a common subchannel. The problem is to determine which subchnnels should be associated to which user. Some authors have already published papers about this problem [4], [5], [6], [10]. However, algorithms proposed in all of these papers do not allow subchannel sharing among different users. In [5] there is an explicit theorem which states that "maximum" data rate of a multiuser OFDM system is achieved only when a subchannel has been used by a single user.

Therefore, in this paper we focus on the development of a new suboptimal loading algorithm for a multiuser OFDM based wireless networks that maximizes the overall data rate while achieving proportional fairness amongst users under a users' QoS requirements and a total power and bits constraints on each subchannel.

The algorithm has two goals:

- assignment of subchannels to users;

- distribution of transmitted signal power and bits among subchannels in such a way that overall data rate of the system is maximized.

The organization of the paper is as follows. Section II introduces a system model for a multiuser environment. Section III describes a new loading algorithm, proposed by the authors, and some of its modifications. In section IV simulation results are presented. Conclusion of this paper is given in section $\mathrm{V}$.

\section{MULTIUSER ENVIRONMENT}

In the downlink transmission of the multiuser OFDM wireless system analyzed in this paper, modulated signals on a number of subcarriers for multiple users are all summed together and they are transmitted through a channel with fading. A block diagram of the downlink transmission system of the multiuser OFDM system is depicted in Fig. 1.

Let $M$ be the number of users in the system. In the figure, $d_{j, i}$ denotes a set of data symbols for the $j$-th user on $i$-th subcarrier, and $s_{j}=\left[s_{j, 1}, s_{j, 2}, \ldots, s_{j, N}\right]$ represents the optimal transmitted power vector of some user $j$. Vector of data rates is 
denoted as $R_{j}=\left[R_{j, 1}, R_{j, 2}, \ldots, R_{j, N}\right] . B$ is the total bandwidth equally divided into $N$ orthogonal subchannels. The bandwidth of a subcarrier signal becomes $B_{m}=B / N$, for all $m$. We assume that all subchannels are narrowband so they can be modeled as Rayleigh flat channels.

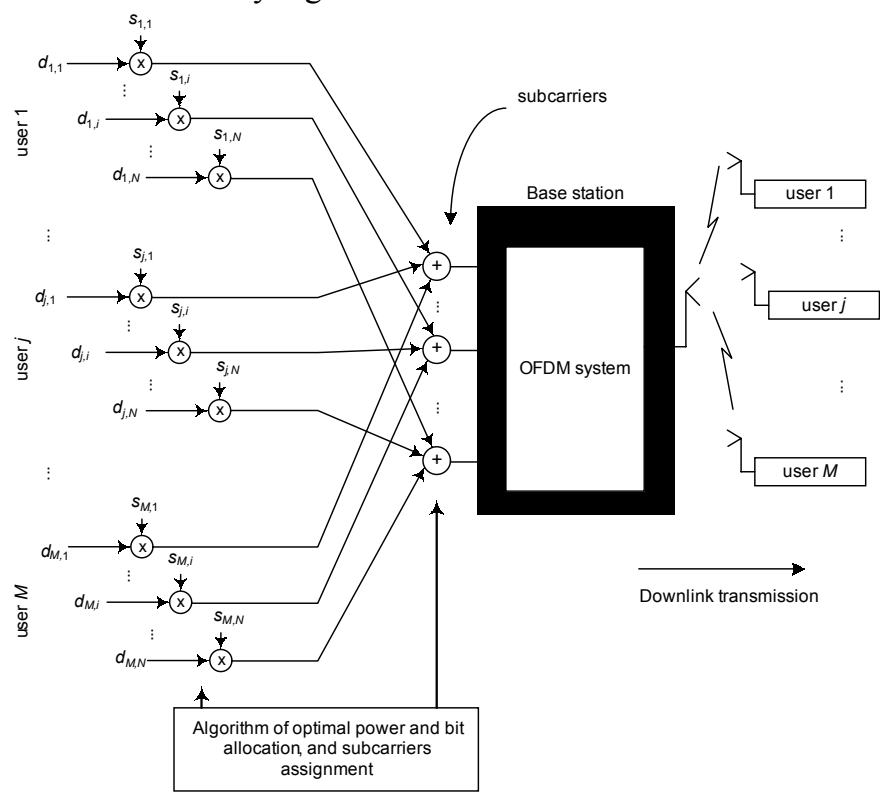

Fig. 1. Block diagram of the downlink OFDM transmission system with adaptation algorithm

Since we allow sharing subchannels among many users then each subchannel in the multiuser OFDM system can be simple considered as a small frequency division multiple access (FDMA) system. In this case, interference between different users' signals exists. From that reason, we have defined a random variable $\omega_{j, m}$ that represents the interference level between modulated users' signals on the subcarrier $m$. Note that the interference level of all users' signals on the same subcarrier is identical, and it will be denoted as $\omega$. This factor controls the power level in order to improve the performance of interference limited communications systems such as OFDM [15] as well. In [15], bounds for interchannel interference for an OFDM system are derived.

In addition, background additive white Gaussian noise (AWGN) with zero mean, variance $\sigma^{2}$ and power spectral density $N_{0}$ is assumed. Since the total bandwidth $B$ is equally divided into $N$ orthogonal subbands then the noise variance can be written as $\sigma^{2}=N_{0} B_{m}=N_{0} B / N$. Finally, the total noise at the receiver's input coming from subchannel $m$ for $i$-th user can be mathematically expressed as

$$
n_{i, m}=\sum_{j=1, j \neq i}^{M} \rho_{j, m} \lambda_{j, m} s_{j, m} \omega_{j, m}+\sigma^{2}
$$

where $\rho_{j, m}$ is an indicator whether user $j$ is transmitting power through subchannel $m$ ( $\rho_{j, m}$ equals to 1$)$ or not $\left(\rho_{j, m}\right.$ equals to $0), \lambda_{j, m}$ is the channel gain of user $j$ on subchannel $m$, and $s_{j, m}$ is the amount of power transmitted by user $j$ through subchannel $m$.

Consequently, the received signal-to-noise ratio (SNR) for the $i$-th user's $m$ - $t$ h subcarrier signal can be written as

$$
\left(\frac{S}{N}\right)_{i, m}=\frac{s_{i, m} \lambda_{i, m}}{\sum_{\substack{j=1 \\ j \neq i}}^{M} \rho_{j, m} \lambda_{j, m} s_{j, m} \omega_{j, m}+N_{0} B_{m}}
$$

\section{PROPOSED LOADING ALGORITHM}

In this paper we assume that the OFDM based transmission system implemented in base station has information about the channels of all users in the system.

In the rate adaptive technique consideration, subcarrier and power allocation have to be carried out jointly to achieve the optimal or suboptimal solution. In the proposed scheme each subcarrier could be allocated to more than one user at any instant of time.

In order to formulate the data rate maximization problem, we will first represent the data rate of the multiuser wireless OFDM system using expression (2). Assuming users' request on QoS expressed by the parameter $\gamma_{j, m}$, the SNR for the $j$-th user in the $m$-th subchannel is bounded by

$$
\gamma_{j, m} \leq\left(\frac{S}{N}\right)_{j, m}
$$

Furthermore, the total data rate, in the multiuser OFDM system, is viewed as the sum of all the users' subcarriers' data rate, and may be represented as

$$
R=\frac{B}{N} \sum_{j=1}^{M} \sum_{m=1}^{N} \log _{2}\left(1+\left(\frac{S}{N}\right)_{j, m}\right)
$$

and the optimization problem can be formulated as follows,

$$
\max _{s_{j, m}, C_{j}} \sum_{j=1}^{M} \sum_{m \in C_{j}} \log _{2}\left(1+s_{j, m} \frac{\lambda_{j, m}}{n_{j, m}}\right)
$$

subject to $\sum_{m=1}^{N} \sum_{j=1}^{M} s_{j, m} \leq S_{\text {total }}$

$s_{j, m} \geq 0$ for all $j, m$

$C_{1}, C_{2}, \ldots, C_{M}$ are not all disjoint

$C_{1} \cup C_{2} \cup \ldots \cup C_{M}=\{1,2, \ldots, N\}$

where Stotal is the overall available power, and $C_{j}$ is the set of all the subcarriers allocated to the $j$-th user. Some fairness among the users on subcarrier are defined through parameter $\lambda_{j, m \text {. }}$

Therefore, to make the maximization problem be tractable, we divide the problem into two steps as follows.

\section{A. Subcarrier assignment to users}

For the first step we assign the subcarrier to a set of users to maximize the feasible data rate for that subcarrier. If user $j$ demands a minimum acceptable SNR (Signal-to-Noise Ratio) on subchannel $m$ denoted by $\gamma_{j, m}$, than the strategy for sharing the subchannel is found from the following theorem.

Theorem 1: $k$ users can share the same subchannel $m$ with required SNR value $\gamma_{j, m}$ only if 


$$
\sum_{j=1}^{k} \frac{\rho_{j, m} \omega_{j, m}}{\rho_{j, m} \omega_{j, m}+\frac{1}{\gamma_{j, m}}}<1
$$

Proof: See the Appendix.

In an OFDM system it is highly probable that a subchannel could be used by most of the users. Every user calculates which subchannel is suitable for him. If he can transmit even one bit in a subchannel, he declares his right on that subchannel usage. In order to satisfy the constraint given in Theorem 1 some users will not be given access to particular subchannels. There are two options. First, in a situation where users have an equal amount of required SNR, i.e., $\gamma$, a practical solution is to find the user with the smallest channel gain on that subchannel. The user is then not allowed to use that subchannel. Second, in the case when each user requires different $\gamma$ in a subchannel, the best solution is probably to "turn off" the user which requires the smallest $\gamma$.

\section{B. Power and bits loading to subcarriers}

For the second step of our loading algorithm, we determine the amount of transmit power to be allocated to the subcarriers in order to maximize the overall data rate. The method of transmit power allocation that maximizes the total system data rate can be found by using Lagrange multiplier technique, [12].

If we define the Lagrangian as

$$
L=\frac{B}{N} \sum_{j=1}^{M} \sum_{m=1}^{N} \log _{2}\left(1+s_{j, m} \frac{\lambda_{j, m}}{n_{j, m}}\right)-\alpha\left(\sum_{m=1}^{N} \sum_{j=1}^{M} s_{j, m}-S_{\text {total }}\right)
$$

where $\alpha$ is the Lagrange multiplier. Solution for $s_{j, m}$ can be obtained by solving the following equation

$$
\frac{\partial L}{\partial s_{j, m}}=0
$$

Solving (8) for all $j$ and $m$ we will get the optimal transmit power vector. Note that the transmit power adaptation method is water-filling [12] over the subcarriers in multiuser OFDM system.

Unfortunately, since there is no explicit method to calculate water-filling level for every symbol period, we have to bypass this numerical method. Therefore, to avoid computational complexity in the water-filling transmit power adaptation method we will adopt a simple equal power allocation method from [13] that in comparison with waterfilling method yields some marginal performance difference. Equal power allocation method gives approximately 2\% less overall data rate in comparison to water-filling method [14]. In our equal power allocation method, the total transmit power is equally distributed among subcarriers after the subcarrier assignment for users has been performed according to the Theorem 1. The power assigned to each subcarrier is divided between users who can share that subcarrier in the following manner. Each user will receive a level of power proportionally to its channel gain in comparison to the other users' power gains on that subcarrier. The proposed equal level allocation strategy for user $j$ that shares subcarrier $m$ can be expressed as

$$
s_{j, m}=\frac{S_{\text {total }}}{N}\left(\lambda_{j, m} / \sum_{k=1}^{M}\left(\lambda_{k, m} \rho_{k, m}\right)\right)
$$

where $S_{\text {total }}$ is the overall available power.

The number of bits per subchannel $m$ for user $j$ is calculated according to the Shannon's information capacity theorem, i.e.

$$
R_{j, m}=\left\lfloor\log _{2}\left(1+\frac{s_{j, m}}{n_{j, m}} \lambda_{j, m}\right)\right\rfloor
$$

where $\lfloor x\rfloor$ denotes a biggest integer that is less or equal to the argument $\mathrm{x}$.

\section{1) Constraint on number of bits in subchannels}

In this algorithm we have limited the number of bits in each subchannel to some value (e.g. num-bits). This value depends on a modulation techniques used in the transmission system, i.e. the modulation technique which can carry the most bits per OFDM subsymbol determines this value. So, upon termination of the algorithm (steps A and B), we should check if this is still satisfied in each subchannel. If not, we have to find the user that transmits the greatest number of bits on that subchannel and subtract one bit from his bit stream. This must be done in order not to favor any user on the subchannel. This procedure is repeated until the number of bits in each subchannel is less or equal to num-bits.

The structured chart of the proposed algorithm is given below.

First step: subcarrier assignment to users.

1. Initialize: set of all subcarriers $C=\{1,2, \ldots, N\}$; $\rho_{j, m}=1, \lambda_{j, m}$ and $\gamma_{j, m} \forall j=1$ to $M$ and $\forall m=1$ to $N$;

2. $\forall j=1$ to $M, C_{j}=\varnothing, s_{j}=0$

3. For $m=1: N$

(a) set $M$ to maximum value

(b) calculate

$$
\Gamma=\sum_{j=1}^{M} \frac{\rho_{j, m} \omega_{j, m}}{\rho_{j, m} \omega_{j, m}+\frac{1}{\gamma_{j, m}}}
$$

(c) If $(\Gamma>1)$ then

(c.1) If $\gamma_{j}$ are all equal then sort $\lambda_{j, m}$ in descending order for $j=1$ to $M$ and set

$\rho_{M, m}=0$ and $M \leftarrow M-1$. Go to step 3.(b).

(c.2) if $\gamma_{j}$ are not equal then sort $\gamma_{j, m}$ in descending order for $j=1$ to $M$ and set

$\rho_{M, m}=0 \quad$ and $M \leftarrow M-1$. Go to step 3.(b).

(d) Set $C_{j}=C_{j} \cup\{m\}$ for users $j=1: M$ who satisfied step (3.b).

End of loop;

4. End of first step.

Second step: bit and power allocation in order to maximize the overall data rate.

5. Calculate (1), (9) and (9a) $\forall j=1$ to $M$ and $\forall m=1$ to $N$

6. For $m=1: N$ 
(6.1) Calculate $R_{m}$ as sum of $R_{j, m} \forall j=1$ to $M$.

(6.2) If $R_{m} \geq$ num_bits then find user with maximum $R_{j, m} \forall j=1$ to $M$ and set the data rate of that user to $R_{j, m} \leftarrow R_{j, m}-1$.

Repeat this step until $R_{m}$ is not less or equal num_bits.

End of loop.

7. Calculate the overall data rate as

$$
R=\frac{B}{N} \sum_{j=1}^{M} \sum_{m=1}^{N} R_{j, m}
$$

8. End of second step and end of algorithm.

\section{SIMULATION RESULTS}

In this section, we present the simulation results of our algorithm in terms of the average data rate or the number of bits per OFDM symbol. To obtain the average value for the number of bits per OFDM symbol we simulated 1000 independent trials, each with a different channel matrix. Also, a comparison of our loading algorithm with one proposed in [5] is done in an environment with three users. Simulation is performed for an OFDM system with 32 subchannels. Furthermore, the number of bits in each subchannel is limited to 12 . Values of interference factor $\omega$ were chosen randomly. The average SNR is defined as $S_{\text {total }} / \sigma^{2}$.

The average number of bits per OFDM symbol versus average SNR for the algorithm that we propose (with $\omega=0.01$ and 0.1 ) and algorithm Jang-Lee (with $\mu=0.1$, [5]) is shown in Fig. 2.

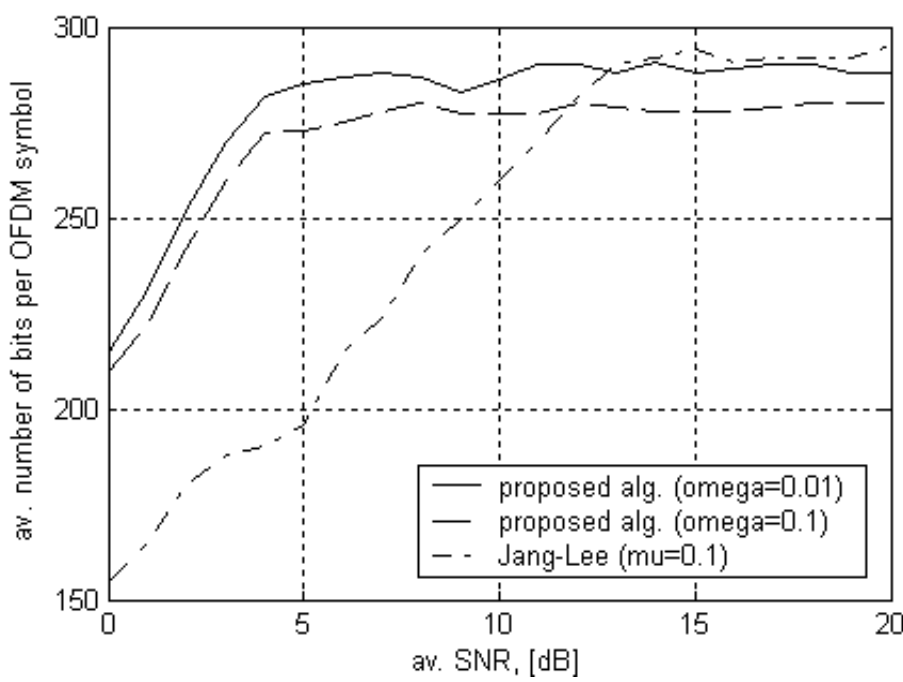

Fig. 2. The number of bits per OFDM symbol versus average SNR for proposed algorithm (the same $\gamma$ requirements) and algorithm JangLee

All of three users have an equal amount of required $\gamma$, i.e. $\gamma$ $=7 \mathrm{~dB}$. It is evident (Fig. 2.) that the proposed algorithm shows better results for all SNR values less than $12 \mathrm{~dB}$. Under these circumstances each subchannel is shared among many users, i.e. the proposed algorithm loads each subchannel up to a maximum, using condition (6) and the limitations on power and bits. In comparison with our algorithm, algorithm JangLee assigns a subcarrier to only one user (the one with the best channel gain on that subcarrier). Sometimes the channel gain on that subchannel has a low value, but greatest in comparison with other values, and the number of bits assigned to those subcarriers is not set to a maximum.

Situation where three users $(1,2$ and 3$)$ require $\gamma$ of 5,10 and $15 \mathrm{~dB}$, respectively, is shown in Fig. 3.

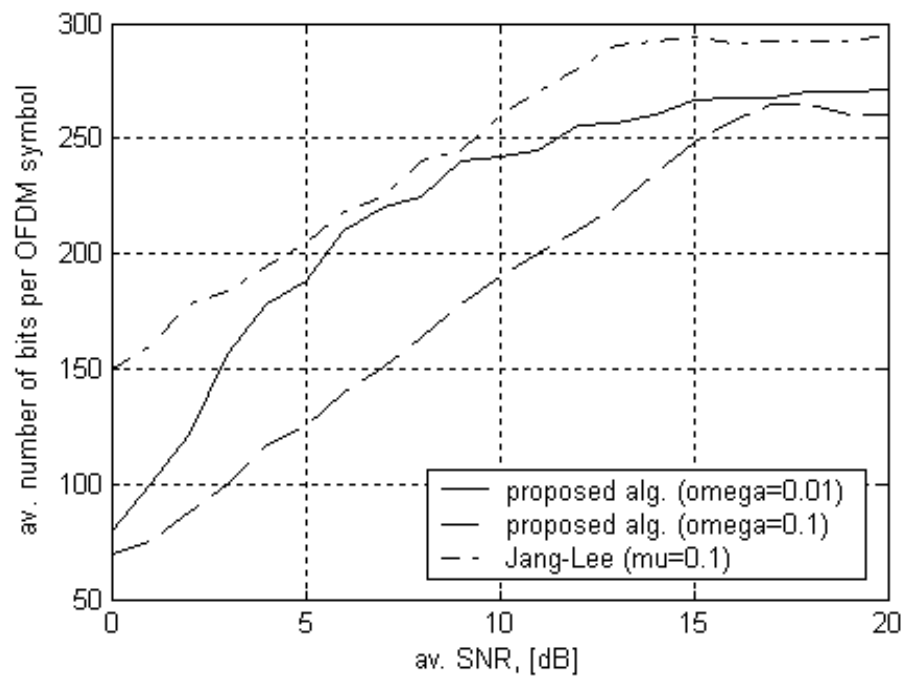

Fig. 3. Number of bits per OFDM symbol versus SNR for proposed algorithm (the different $\gamma$ requirements) and algorithm Jang-Lee

If condition (6) is not satisfied than we use SNR or $\gamma$ as a criterion for "turning off" a user in a particular subchannel. For this case, algorithm Jang-Lee shows better results than our algorithm for all the values of average SNR. Furthermore, it is visible that the data rate achieved by our algorithm depends mostly on interference factor $\omega$.

Fig. 4 depicts some bounds for variables $\omega$ and $\gamma$. Simulation is performed in an environment with three users. Values for factor $\omega$ are $0.01,0.08$ and 0.1 .

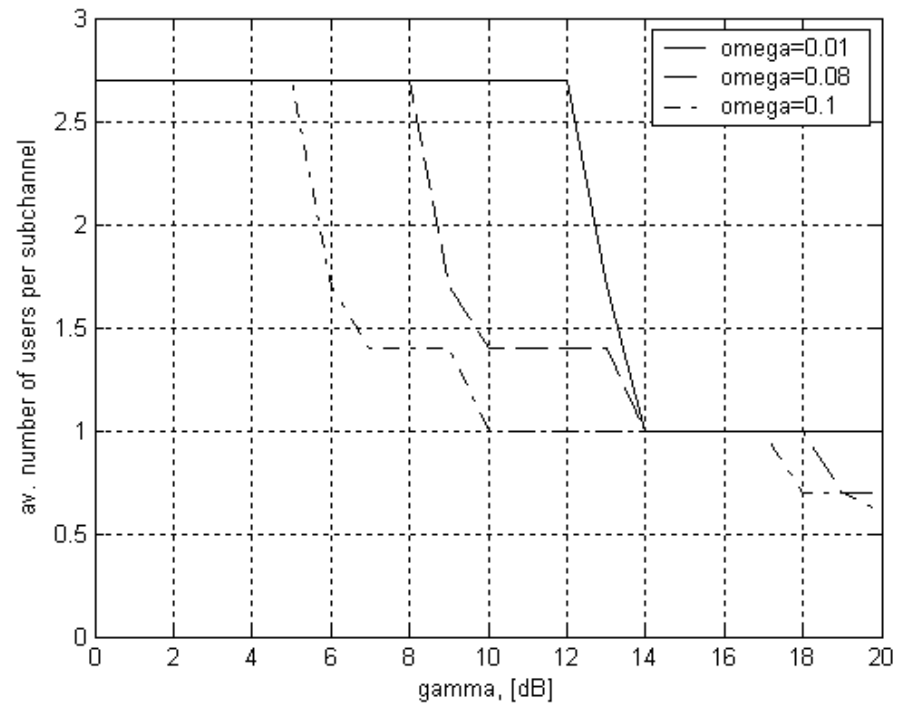

Fig. 4. Average number of users per subchannel with const. factor $\omega$

For low values of $\gamma$, almost every subchannel is shared by two users, no matter how big factor $\omega$ is. Furthermore, for $\gamma$ 
greater than or equal to $14 \mathrm{~dB}$, each subchannel belongs to only one user. This situation can be seen as a confirmation of the theorem stated in [5]. From this we can conclude that the algorithm proposed in [5] can be seen as a special case of our algorithm. As we can see, if variable $\gamma$ is set to a high value, subchannels will not be shared.

The average number of bits per OFDM symbol versus the number of users (average SNR $=8 \mathrm{~dB}$ and $\gamma=7 \mathrm{~dB}$ were taken for all users) is shown in Fig. 5.

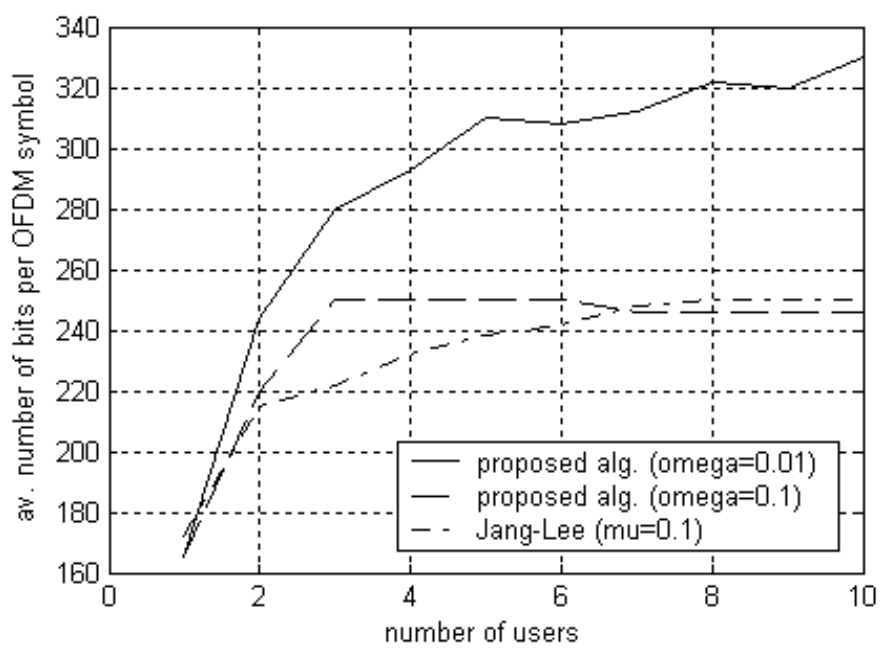

Fig. 5. Number of bits per OFDM symbol versus number of users

From the Fig. 5 it is obvious that the average number of bits per OFDM symbol for the proposed method increases as the number of users increases. Some small variations (i.e. decrease) of the number of bits per OFDM symbol are a consequence of the state of the channel. Generally, for the proposed loading algorithm, since more than one user can share each subcarrier, the average SNR for each subcarrier increases as the number of users increases, and the total data rate increases as a result.

The authors of [11] and [4] have show similar results, i.e. the data rate of the multiuser system, with the optimal power allocation algorithm, increases as the number of users increase.

\section{CONCLUSION}

In this paper we have proposed an suboptimal subcarrier, bit and power loading algorithm for wireless multiuser OFDM environment that maximizes the data rate or number of bits per OFDM symbol in downlink transmission. The proposed algorithm belongs to a class of algorithms with constraints. It means that we have performed simulations with constraints on the transmitter power and the number of bits per each subcarrier. While formulating the problem in multiuser OFDM systems, we have allowed a subcarrier to be simultaneously shared by multiple users, and the power and bit allocation algorithm has been derived via two steps: subcarrier assignment to users, and bit and power allocation for subcarriers. Also, we have defined two variables: the quality of service (QoS) factor, $\gamma$, which defines the signal to total noise ratio at the receiver side required by the user, and interference factor, $\omega$, which models the signal interference between users on the same subcarrier. However, the proposed algorithm requires the complete channel information including the level of interference between users. This information is obtained by reverse channel (from users to base station). In this paper we have not analyzed this channel, but we have taken in to account that this channel is error free.

We have compared our loading algorithm with the algorithm Jang-Lee proposed in [5]. The authors of [5] found that the data rate could be maximized if each subcarrier in OFDM system is assigned to only one user who has the best channel gain. Our simulation results show that it is possible to achieve a higher data rate than is achieved by the Jang-Lee algorithm. This is possible if variables $\gamma$ and $\omega$ are properly chosen. Although this paper considered the situation when no interference cancellation technique is used. It means that the proposed algorithm can also be used in conjunction with some multiuser diversity methods, [11], in order to control values of variable $\omega$.

We have proved that algorithm proposed in [5] can be observed as a special case of our algorithm. Results show that the numbers of bits per OFDM symbol (date rate) for the proposed loading algorithm considerably increase as the number of users increases. Also, because of small amount of logarithm operations the proposed algorithm is very well suited for high speed data transmission networks over cooper pair lines. However, our loading algorithm leaves open a question about quality of service for each user, especially about the demanded bit rate.

\section{Appendix: Proof of Theorem 1}

In order to find the transmit power allocation rule that maximizes the data rate of a multiuser OFDM system, we solve the subcarrier assignment first. Theorem 1 states that $M$ users can share the same subchannel $m$ with required SNR value $\gamma_{j, m}$ only if it satisfies

$$
\sum_{j=1}^{M} \frac{\rho_{j, m} \omega_{j, m}}{\rho_{j, m} \omega_{j, m}+\frac{1}{\gamma_{j, m}}}<1
$$

Signal to noise ratio (SNR) for $i$-th user on $m$-th subcarrier can be written as

$$
\left(\frac{S}{N}\right)_{i, m}=\frac{s_{i, m} \lambda_{i, m}}{n_{i, m}+N_{0} B_{m}}=\frac{s_{i, m} \lambda_{i, m}}{\sum_{\substack{j=1 \\ j \neq i}}^{M} \rho_{j, m} \lambda_{j, m} s_{j, m} \omega_{j, m}+N_{0} B_{m}}
$$

Introducing user's request on SNR, the problem is then formulated as

$$
\left(\frac{S}{N}\right)_{i, m}=\frac{s_{i, m} \lambda_{i, m}}{\sum_{\substack{j=1 \\ j \neq i}}^{M} \rho_{j, m} \lambda_{j, m} s_{j, m} \omega_{j, m}+N_{0} B_{m}} \geq \gamma_{i, m}
$$

The one of power vectors satisfying condition (A.3) is obtained if 


$$
\begin{gathered}
\frac{s_{i, m} \lambda_{i, m}}{\sum_{\substack{j=1 \\
j \neq i}}^{M} \rho_{j, m} \lambda_{j, m} s_{j, m} \omega_{j, m}+N_{0} B_{m}}=\gamma_{i, m} \\
\frac{s_{i, m} \lambda_{i, m}}{\gamma_{i, m}}-\sum_{\substack{j=1 \\
j \neq i}}^{M} \rho_{j, m} \lambda_{j, m} s_{j, m} \omega_{j, m}=N_{0} B_{m}
\end{gathered}
$$

This yields to the matrix formulation $\mathbf{D S}=N_{0} B_{m} \mathbf{I}$ where

$$
\mathbf{D}=\left(\begin{array}{cccc}
\frac{\lambda_{1, m}}{\gamma_{1, m}} & -\rho_{2, m} \lambda_{2, m} \omega_{2, m} & \cdots & -\rho_{M, m} \lambda_{M, m} \omega_{M, m} \\
-\rho_{1, m} \lambda_{1, m} \omega_{1, m} & \frac{\lambda_{2, m}}{\gamma_{2, m}} & \cdots & -\rho_{M, m} \lambda_{M, m} \omega_{M, m} \\
\vdots & \vdots & \ddots & \vdots \\
-\rho_{1, m} \lambda_{1, m} \omega_{1, m} & -\rho_{2, m} \lambda_{2, m} \omega_{2, m} & \cdots & \frac{\lambda_{M, m}}{\gamma_{M, m}}
\end{array}\right)
$$

$\mathbf{S}=\left[s_{1, m}, s_{2, m}, \ldots, s_{M, m}\right]^{T}$ is the optimal power vector, and $\mathbf{I}$ is the unity matrix. Doing two elementary matrix operations (subtracting first row from the next, and starting from the second row, multiply each row with $\frac{\rho_{j, m} \omega_{j, m}}{\rho_{j, m} \omega_{j, m}+\frac{1}{\gamma_{j, m}}}$ and add it to the first row) the matrix form will be reduced. Diagonal elements and elements in the first column will remain. The equation with parameter $s_{I, m}$ is

$$
\left(\rho_{1, m} \omega_{1, m}+\frac{1}{\gamma_{1, m}}\right) \lambda_{1, m}\left(1-\sum_{j=1}^{M} \frac{\rho_{j, m} \omega_{j, m}}{\rho_{j, m} \omega_{j, m}+\frac{1}{\gamma_{j, m}}}\right) s_{1, m}=N_{0} B_{m}
$$

Power $s_{l, m}$ must be positive and this yields to the following condition

$$
\sum_{j=1}^{M} \frac{\rho_{j, m} \omega_{j, m}}{\rho_{j, m} \omega_{j, m}+\frac{1}{\gamma_{j, m}}}<1
$$

which completes the proof of the theorem.

\section{REFERENCES}

[1] Jiho Jang, Kwang Bok Lee, Yong-Hwan Lee, "Transmit power and bit allocations for OFDM systems in a fading channel", In Proc. of IEEE Global Communications Conference 2003 (GLOBECOM 2003), Dec. 2003.

[2] Achankeng Leke, John M. Chioffi, "A maximum rate loading algorithm for discrete multitone modulation sytems", In Proc.of IEEE Globecom 1997, p.p. 1514-1518, Nov 1997.

[3] Krongold, B. S. Ramchandran, K. Jones, D.L., “ Computationally Efficient Optimal power Allocation Algorithms for Multicarrier Systems", IEEE Trans. on Communications, vol. 48 (1), p.p. 23-27, Jan 2000.

[4] Cheong Yui Wong, Roger S. Cheng, Khaled Ben Letaief and Ross D. Murch, "Multiuser OFDM with Adaptive Subcarrier, Bit, and Power Allocation", IEEE Journal of Selected Areas in Comm., vol. 21 (2), p.p. 171-178, Feb 2003.

[5] Jiho Jang and Kwang Bok Lee, "Transmit Power Adaptation for Multiuser OFDM systems", IEEE Journal of Selected Areas in Comm., vol. 17 (10), p.p. 1747-1758, Oct 1999.
[6] Wonjong Rhee, John M. Cioffi, "Increase Capacity of Multiuser Ofdm System Using Dynamic Subchannel Allocation", In Proc. of Conference VTC 2000, Tokyo.

[7] M. Engels, Wireless OFDM systems: how to make them work?, Boston: Kluwer Academic Publishers, 2002.

[8] D. Hughes-Hartogs, "Ensemble modem structure for imperfect transmission media," U.S. Patents Nos. 4,679,227 (July 1987), 4,731,816 (March 1988), and 4,833,706 (May 1989).

[9] P. S. Chow, J. M. Cioffi, and J. A. C. Bingham, "A practical discrete multitone transceiver loading algorithm for data transmission over spectrally shaped channels," IEEE Trans. Commun., vol. 43, pp. 773775, Feb./Mar./Apr. 1995.

[10] C. Y. Wong, C. Y. Tsui, R. S. Cheng, and K. B. Letaief, "A real-time subcarrier allocation scheme for multiple access downlink OFDM transmission," In Proc. IEEE Veh. Tech. Conf. VTC'99), p.p. 1124-1128. Nov. 1999.

[11] R. Knopp and P. A. Humblet, "Information capacity and power control in single-cell multiuser communications," in Proc. IEEE Int. Conf. Communications 1995 (ICC'95), Seattle, WA, June 1995, p.p. 331-335.

[12] T. M. Cover and J.A. Thomas, Elements of Information Theory, New York, Willey, 1991.

[13] E. Biglieri, J. Proakis, and S. Shamai, "Fading channels: Information theoretic and communications aspects," IEEE Trans. Inform. Theory, vol. 44, pp. 2619-2692, Oct. 1998.

[14] A. Leke and J. M. Cioffi. "Transmit Optimization for Time-Invariant Wireless Channels Utilizing a Discrete Multitone Approach," In Proc. IEEE ICC'97, Montreal, June 1997, pp. 954-958.

[15] Y. (G.) Li, L. J. Cimini Jr., "bounds on the Interchannel Interference of OFDM in Time-Varying Impairments," IEEE Trans. Commun., vol. 49, pp. 401-404, March 2001.

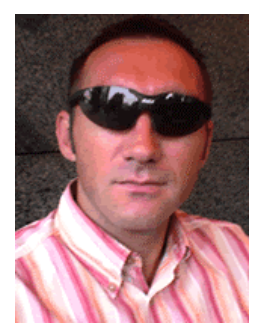

Zeljko Ilic (M'02) received the B.S., M. S. and $\mathrm{Ph} . \mathrm{D}$. degrees in electrical engineering in 1996, 1989 and 2004, respectively, from the Faculty of Electrical Engineering and Computing, University of Zagreb at Zagreb, Croatia.

Currently, he is working as an assistant at the Department of Telecommunications, Faculty of Electrical Engineering and Computing at the University of Zagreb. His interests include signal processing in communications, information theory and access telecommunication networks. He is author and co-author around 20 papers on these topics published in books, journals and conferences.

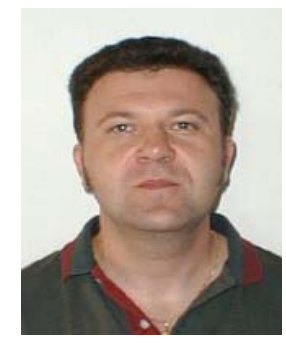

Alen Bazant (M'97) received the B.S., M. S. and $\mathrm{Ph} . \mathrm{D}$. degrees in electrical engineering in 1985, 1990 and 1998, respectively, from the Faculty of Electrical Engineering and Computing, University of Zagreb at Zagreb, Croatia.

He began to work on the Faculty of Electrical Engineering and Computing (FER) at January 1987 as a departmental assistant on the Department of Telecommunications. In 1989 he began to work in a company Nikola Tesla (present Ericsson Nikola Tesla) in Zagreb. Upon receiving masters degree he was shortly with Croatian Telecomm (former HPT), and got back to FER where he has been employed as an assistant involved in teaching and research. In 1993 he was with two companies in Zagreb, Optima O.S.N. and TIS d.d. His primary occupation in these companies was related to software engineering. In $1994 \mathrm{he}$ had returned to FER and began a Ph.D. study. During 1995 and 1996 he spent six months in Ericsson New Zealand in Wellington working on problems related to mobile telephony. In May 1996 he returned to FER. Since 2000 he has been assistant professor. He is a coauthor of one textbook. His research interests are in transmission media characteristics and analyses, modulations and line coding, in multiple access technologies used in local area networks and in technologies related to lowest two layers of OSI reference model. 